\title{
Fibroblast Growth Factor 23 and Carotid Artery Intima Media Thickness in Children with Chronic Kidney Disease
}

\author{
Moftah Mohammed Rabeea', Nayera Mahmoud El-Akkad', Mohammed Yousry Shahin'², Mustafa \\ Fadel M. Sonbol' ${ }^{3}$, Hazem El- Sayed M. Abd El Wahab'
}

1. Pediatric department Al-Azhar University

2. Clinical Pathology department Al-Azhar University

3. Radiology department Al-Azhar University

\begin{abstract}
Background

The cause of early-accelerated atherosclerosis development observed in chronic kidney disease (CKD) is not fully understood. The determination of the relationship between the serum or blood levels of fibroblast growth factor 23 (FGF 23) and carotid artery intima media thicknesses (CIMT) and development of endothelial dysfunction lend support to the possibility that FGF-23 plays a role in the development of atherosclerosis in CKD.
\end{abstract}

\section{Aim of Work}

To assess the circulating FGF23 as a marker of vascular stiffness in children with CKD on conservative treatment regardless the etiology and to correlate its level with carotid artery intima media thickness as detected by ultrasonography.

\section{Patients and Methods}

This is a prospective case control study was done on forty cases with CKD on conservative treatment (stage II - III and IV) regardless the etiology. Also twenty apparently healthy children age and sex matched with cases were included as a control group. After complete clinical evaluation of cases and controls including weight, height, body mass index, blood pressure and all body systems, FGF23 was measured in serum and carotid artery intima media thicknesses (CIMT) was measured as a marker of atherosclerosis using ultrasonography.

\section{Results}

CIMT measurements and FGF-23 levels were significantly higher in patients than controls. There was a positive correlation between FGF23 and both CIMT, CKD stage. On the other hand there was a negative correlation between FGF23 and the level of glomerular filtration rate (GFR).

\section{Conclusion}

FGF-23 may be used as a sensitive and non-invasive indicator of subclinical atherosclerosis in patients with chronic kidney disease where our results showed a positive correlation between FGF23 and CIMT.

\section{Key words}

Chronic Kidney Disease, Fibroblast Growth Factor 23, Carotid Artery Intima Media Thicknesses, Glomerular Filtration Rate.

\section{Correspondence}

Hazem El Sayed Mohammed

Pediatric department, Al-Azhar University, Cairo, Egypt.

Email: hazemsaleh18@yahoo.com

\author{
geget : The Journal of the Egyptian Society of Pediatric Nephrology and Transplantation (ESPNT) \\ geget https://geget.journals.ekb.eg/ \\ Published by ESPNT http://espnt.net/ \\ Cohosted by Egyptian Knowledge Bank https://www.ekb.eg
}




\section{Introduction}

Chronic kidney disease (CKD) describes the gradual loss of kidney function. Kidneys filter wastes and excess fluids from blood, which are then excreted in urine. When chronic kidney disease reaches an advanced stage, dangerous levels of fluid, electrolytes and wastes can build up in the body [1].

Fibroblast growth factor-23 (FGF-23), a 251-amino acid protein synthesized and secreted by osteoblasts and osteocytes, is a potent regulator of serum phosphate levels [2]. Several hereditary and acquired diseases associated with excessive circulating level of FGF23. Hereditary diseases include autosomal dominant, autosomal recessive, and Xlinked hypophosphatemic rickets. Acquired diseases include McCune-Albright syndrome, polyostotic fibrous dysplasia, tumor-induced osteomalacia and epidermal nevus syndrome [3].

Examination of the carotid artery intima media thickness (CIMT) gives every clinician an opportunity to evaluate subclinical alterations in wall structure that precede and predict future cardiovascular clinical events. B-mode ultrasonography is a noninvasive, safe, easily performed, sensitive, relatively inexpensive and widely available method for detection of early stages of atherosclerosis and is accepted as one of the best methods for evaluation of CMIT [4].

\section{Aim of the Work}

To assess the circulating FGF23 as a marker of vascular stiffness in children with CKD on conservative treatment regardless the etiology and to correlate its level with carotid artery intima media thickness as detected by ultrasonography.

\section{Patients and Methods}

This is a prospective case control study was carried out on forty cases with CKD regardless the etiology. Those patients were chosen for the study from the Combined Clinic of Pediatric Nephrology \& Urology during the period from October 2016 to April 2018.

Their ages ranged from 2 to 17 years with mean age of 9.4 \pm 4.7 years. They were 26 males and 14 females. In addition, twenty apparently healthy children age and sex matched with the cases were included as a control group.

Inclusion criteria:

- All cases with CKD stages (II-III and IV) regardless the etiology.

- Staging of CKD in our cases based on the level of GFR according to the classification of (Kidney Disease Improving Global Outcomes (KDIGO) , 2013)

\section{Exclusion criteria:}

- Cases with other systemic diseases or chronic illnesses (comorbidities) other than SLE

- $\quad$ End stage renal disease and patients with CKD who are not regular on follow up and not compliant to medication.

- Patients with neoplastic, metabolic and hereditary diseases that affect multiple systems eg: lipid storage disease, Wilson disease....etc.

\section{Ethical considerations}

- An informed verbal consent was obtained from all parents of patients and control group before getting them involved in the study.

- This study was approved by the ethical committee for researches of both Pediatric Department 12-2018 No 405) and Al-Azhar faculty of medicine (14-12 -2018).

- The steps of the study, the aim, the potential benefits and hazards, all were discussed with the parents of the studied groups.

- Confidentiality of all data was ensured.

- The patients and controls had the right to withdraw from the study at any time without giving any reasons.

For all included cases the following was done:

1. Detailed history especially for: urinary symptoms, history of renal diseases in the family and pertinent medications, family history of hypertension, history of hospital admission due to renal diseases and medications received.

2. Thorough clinical examination including: height, weight, body mass index (BMI), blood pressure and all body systems.

3. Laboratory investigations including:

- CBC by Sysmex KX and CRP.

- Urine analysis \& culture.

- Blood urea, serum creatinine by ELISA.

- Serum calcium, phosphorus, alkaline phosphatase and parathormone hormone by ELISA

- Lipid profile (serum cholesterol and serum triglycerides) by ELISA.

- Serum fibroblast growth factor 23 by ELISA

4. Imaging studies including:

- Carotid artery intima media thickness by ultrasonography for all cases and controls

- Renal U/S for all cases and controls

- MRU, VCUG and Renal scan in selected cases (those with significant findings on U/S)

5. Renal biopsy: For certain cases ( 8 cases) (in whom congenital obstruction of the urinary tract, renal stones and nephrocalcinosis have been excluded)

6. Glomerular filtration rate (GFR) using:

- Kinetic renal scan using diethylene triamine pentaacetic acid (DTPA) (it gives the split function of each kidney) in cases with obstructive uropathy GFR was measured by renal scan( renal scan already done at pediatric urology unit as the study is a combined one)

- Schawrtz formula for all cases (Schawrtz formula , 2009)

GFR $(\mathrm{ml} / \mathrm{min} / 1.73 \mathrm{~m} 2)=(\mathrm{K} \times$ height in $\mathrm{cm}$ or length) / serum creatinine

$\mathbf{K}=0.45$ in term and normal infants during first year of life

$\mathbf{K}=0.55$ in children and adolescent girls

$\mathbf{K}=0.70$ in adolescent boys 
As regard to controls, they were subjected to history taking, clinical evaluation, routine laboratory investigations, abdominal ultrasonography, measurement of carotid artery intima media thickness and serum fibroblast growth factor 23 .

\section{Statistical analysis}

- Comparison between groups was performed using independent sample t-test for quantitative variables and Chi square test or Fisher's exact test for qualitative ones.

\section{Results}

- Paired t-test was used for comparison of creatinine repeated measures.

- Pearson correlation coefficients were calculated to signify the association between different quantitative variables.

○ $\mathrm{P}$ values less than 0.05 were considered statistically significant, and less than 0.01 were considered highly significant.

- Graphs were used to illustrate some information.

- Spearman correlation coefficient test (r): was used to test a positive or negative relationship between two variables.

Table 1: Age \& sex of the studied cases \&controls.

\begin{tabular}{|c|c|c|c|}
\hline & Cases $(\mathbf{n}=\mathbf{4 0})$ & Controls (n=20) & P value \\
\hline Sex $n,(\%)$ & & & 0.17 \\
\hline Male & $26(65 \%)$ & $9(45.0 \%)$ & NS \\
\hline Female & $14(35 \%)$ & $11(55.0 \%)$ & 0.9 \\
\hline Age(years) & $2-17$ & $4-15$ & NS \\
\hline Range & $9.4 \pm 4.7$ & $8.9 \pm 3.5$ & $9.0(5.3-11.8)$ \\
\hline Mean \pm SD & $8.5(6.0-14.0)$ & & \\
\hline
\end{tabular}

Table 1 revealed that there was no significant difference between the two groups as regard age and sex, the prevalence of CKD was more in males $(65 \%)$ more than females $(35 \%)$.

Table 2: Etiology of CKD in the studied cases

\begin{tabular}{|c|c|c|}
\hline Etiology of CKD & Frequency (n=40) & Percentage (\%) \\
\hline Obstructive uropathy & 29 & $72.5 \%$ \\
\hline Lupus nephritis & 4 & $7.5 \%$ \\
\hline Bilateral multiple stones & 3 & $2.5 \%$ \\
\hline Membranous glomerulonephritis & 1 & $2.5 \%$ \\
\hline Severe interstitial nephritis & 1 & $2.5 \%$ \\
\hline FSGS (focal segmental glomerulosclerosis) & 1 & $2.5 \%$ \\
\hline Tlobal \& segmental glomerulosclerosis, moderate chronic & 1 & $\mathbf{1 0 0 \%}$ \\
\hline
\end{tabular}

Table 2 revealed that obstructive uropathy is the most common etiology of CKD in our patients. 
Table 3: Results of GFR in the studied cases according to stages of CKD.

\begin{tabular}{|c|c|c|c|}
\hline Stage of CKD & NO of cases & Percentage (\%) & Level of GFR (ml/min/1.73m2) \\
\hline Stage II & 15 & $37.5 \%$ & $45-62$ \\
\hline Stage III & 15 & $37.5 \%$ & $29-22$ \\
\hline Stage IV & 10 & $25 \%$ & 23 \\
\hline
\end{tabular}

Table 3 revealed that stage II \& III represent $37.5 \%$ of cases for each stage where stage IV represents $25 \%$ of cases.

Table 4: Comparison between cases \& controls regarding FGF23 and CIMT

\begin{tabular}{|c|c|c|c|c|c|c|c|}
\hline & \multicolumn{3}{|c|}{ Cases (N=40) } & \multicolumn{2}{c|}{ Controls (N=20) } & P value \\
\hline & Mean \pm SD & Median & IQR & Mean \pm SD & Median & IQR & \\
\hline FGF23 (pg/ml) & $371 \pm 276$ & 312 & 104 & $34 \pm 7$ & 35 & 27 & $<0.001$ \\
\hline CIMT (mm) & $0.81 \pm 0.3$ & 0.79 & .51 & $0.29 \pm 0.08$ & 0.31 & .21 & $<\mathbf{0 . 0 0 1}$ \\
\hline
\end{tabular}

Table 4 and Figures $(3,4)$ revealed that there were significant differences between cases and controls as regard to FGF23 and CIMT.

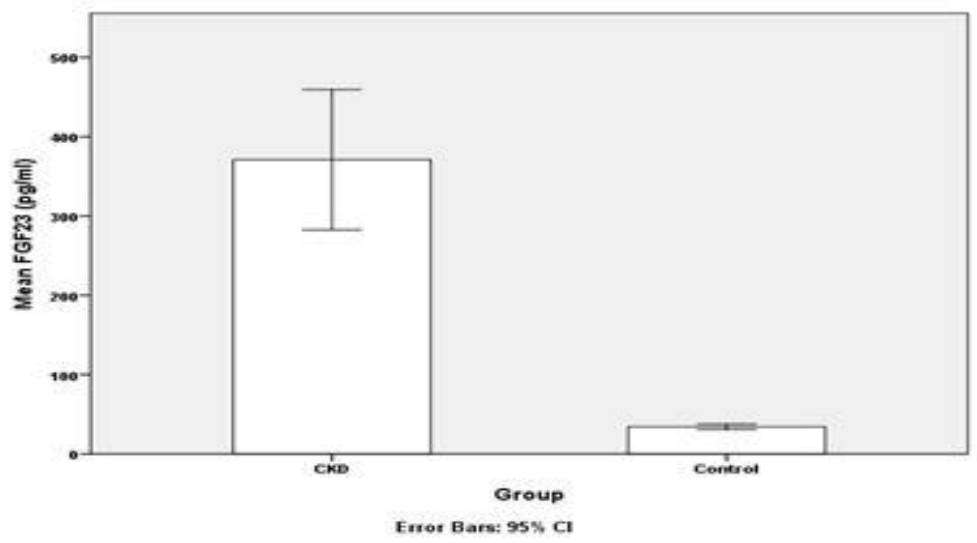

Figure 3: Mean FGF23 level in cases with CKD and controls. Error bars represent the 95\% confidence interval (95\% CI).

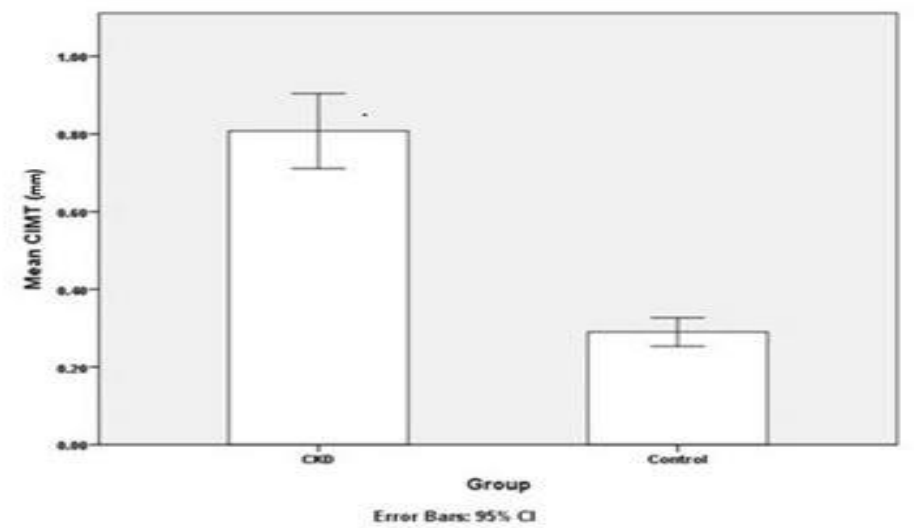

Figure 4: Mean CIMT in cases with CKD and controls. Error bars represent the $95 \%$ confidence interval (95\% CI). 
Table 5: Correlations between FGF23 and other important quantitative variables.

\begin{tabular}{|c|c|c|}
\hline & FGF23 & P-value \\
\hline Variable & Pearson's r & $<\mathbf{0 . 0 0 1}$ \\
\hline CIMT & 0.790 & $<\mathbf{0 . 0 0 1}$ \\
GFR & -0.865 & $<\mathbf{0 . 0 0 1}$ \\
\cline { 2 - 3 } & 0.854 & \\
\hline
\end{tabular}

Table 5 and Figures $(\mathbf{5 , 6}$, and 7) revealed that there were positive correlations between FGF23 and both CIMT, CKD stage and negative correlation between FGF23 and GFR.

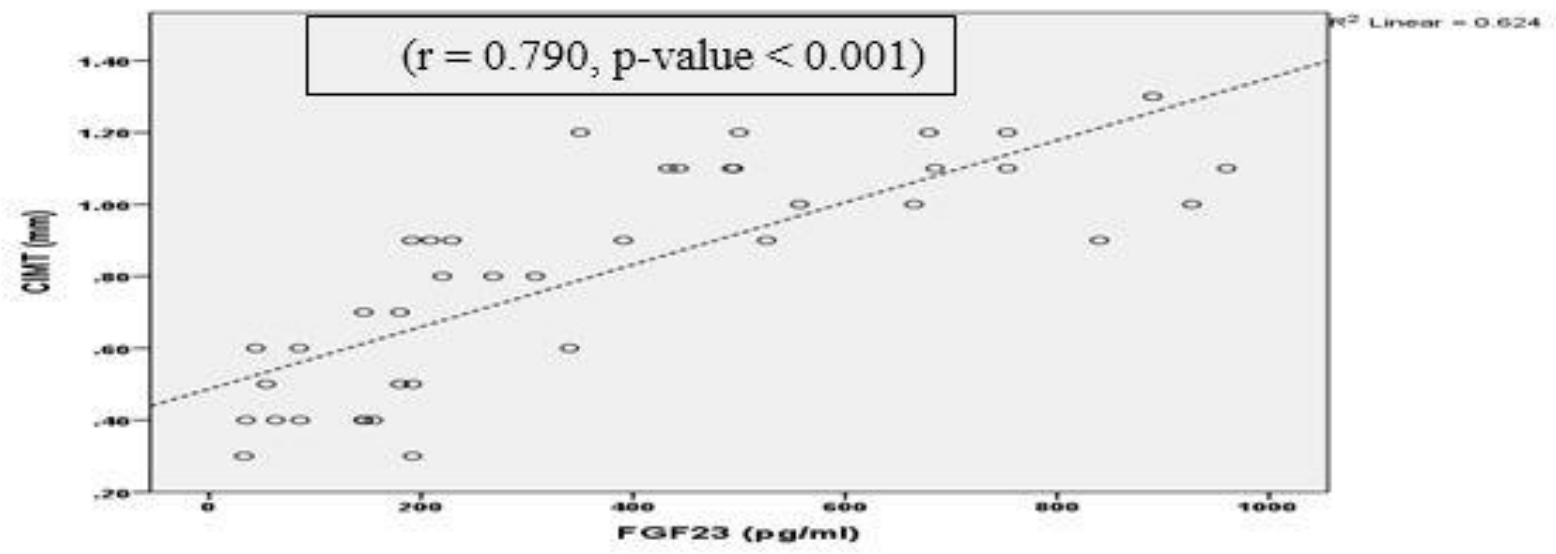

Figure 5: Scatter plot illustrating the correlation between FGF23 and the CIMT. There is a strong positive correlation between FGF23 and the CIMT.

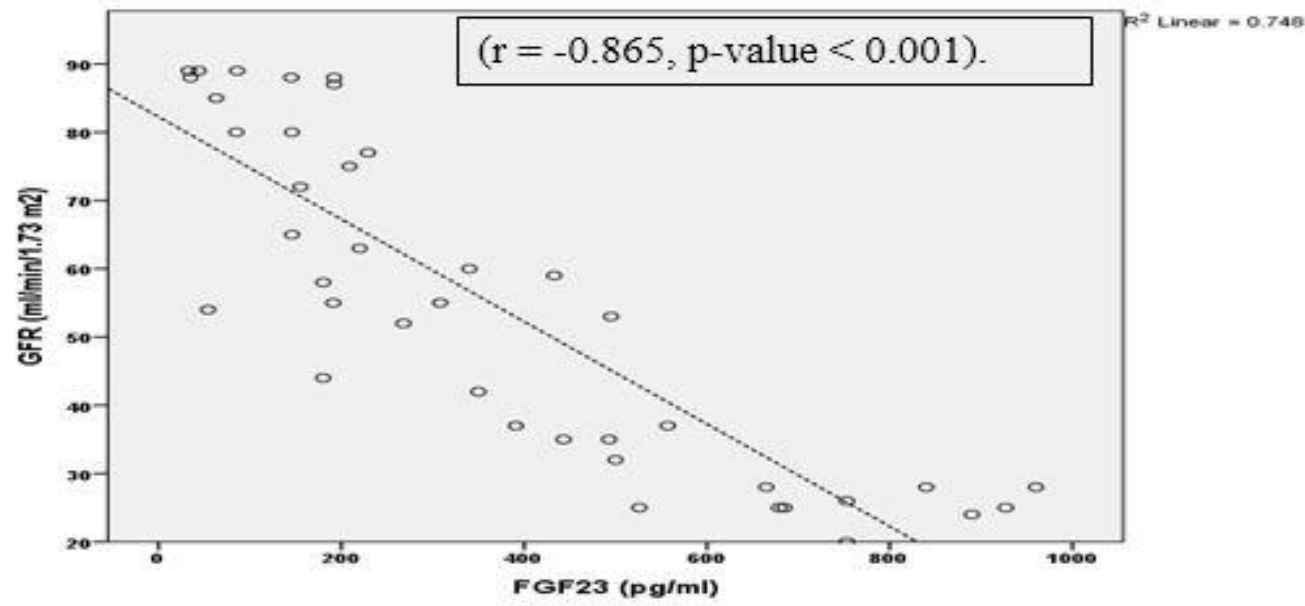

Figure 6: Scatter plot illustrating the correlation between FGF23 and the GFR. There is a strong negative correlation between FGF23 and the GFR. 


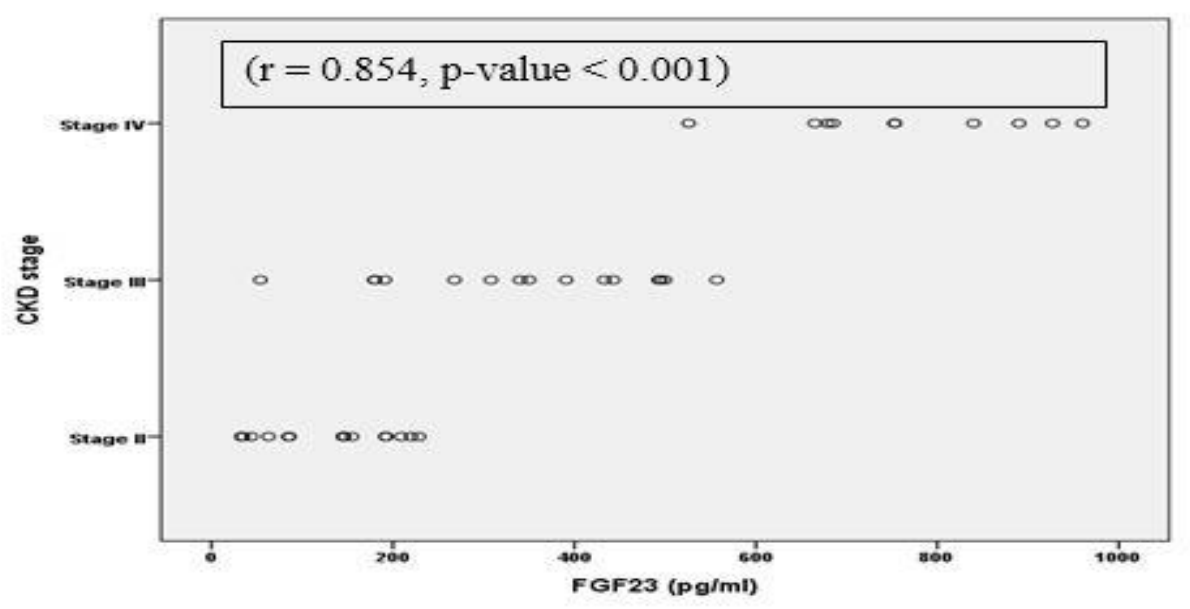

Figure 7: Scatter plot illustrating the correlation between FGF23 and the stage of CKD. There is a strong positive correlation between FGF23 and the stage of CKD.

\section{Discussion}

Patients with CKD develop early in the course of the disease structural and functional alterations of the heart and the vascular tree that represent a major clinical problem in these patients [5]. Laboratory assessment of FGF23 and ultrasonography for those patients was the main scope of our study to detect if there is a relation between FGF23 and CIMT for detection of preclinical atherosclerosis.

As regard to demographic data, our study revealed that the prevalence of CKD was higher in males $(\mathbf{6 5 \%})$ than in females $\mathbf{( 3 5 \% )}$ ). Male preponderance may be explained by higher prevalence of obstructive uropathy in our study which is generally common in boys. This agrees with the study which showed male preponderance in their studies on CKD children on conservative treatment [6] Also in another study, it has been reported that the incidence and prevalence of CKD is greater in males than females because of the higher frequency of congenital abnormalities of the kidney and urinary tract (CAKUT) in males[7]. Regarding the etiology of CKD, obstructive uropathy was the commonest cause of CKD in our study This agrees with the study showed that the commonest cause of CKD was CAKUT, followed by steroid resistant nephrotic syndrome and glomerulonephritis [8]

Our results showed a significant differences between cases and controls as regard to FGF 23.This agrees with the study done on 91 patient with chronic kidney disease. They found that FGF 23 was significantly higher in patients than controls [9]. The present study showed that measurements of CIMT were significantly higher in cases than the control group. These data agree with the Egyptian study done on 40 children with CKD divided into two groups: 20 children had chronic kidney disease on conservative therapy (group I) and 20 children were undergoing regular hemodialysis (group II). Also 20 healthy children were included as a control group (group III) and found that there was a significant increase in CIMT in group I and group II when each group was compared with group III [10].

There was a strong positive correlation between FGF23 and the CIMT. These data agree with the results of Yelmas et al (2015) where the authors found a positive correlation between FGF23 and CIMT and monitoring of serum FGF23 may be a useful non-invasive indicator of subclinical atherosclerosis in patients with CKD.

There was a strong negative correlation between FGF23 and GFR and a strong positive correlation between FGF23 and the stage of (CKD).

Our results are supported by the study done on pediatric CKD patients stage I to $\mathrm{V}$ and it was concluded that FGF23 had a strong negative correlation with eGFR and FGF23 levels rise as the renal function declines [11].

\section{CONCLUSION}

- Fibroblast growth factor 23 can be considered one of the markers of development of endothelial dysfunction and development of preclinical atherosclerosis

- Measurement of CIMT is of value to diagnose the atherosclerosis in CKD patients

- Positive correlation between FGF23 and CIMT indicating that FGF23 plays an important role in the development of vascular calcifications

- Children with CKD are prone to the development of vascular calcifications and becoming more prevalent with the worsening of kidney function as proved by the negative correlation between GFR and the level of FGF23

\section{RECOMMENDATIONS}

- Regular follow up for children with CKD include: clinical and laboratory evaluation as well as imaging studies for early detection of progression in kidney function renal.

- Measurement of CIMT in CKD children to detect early atherosclerotic changes is of significant value starting from stage III and repeated according to the progression (however some cases need to be individualized). 


\section{References}

1- Goldman L: Chronic kidney disease. In GoldmanCecil Medicine 2016. 25th ed. Philadelphia.

2- Sarmento-Dias M, Santos-Araujo C, Poínhos R, Oliveira B, Silva IS, Silva LS, Sousa MJ, Correia F, Pestana M: FGF 23 is associated with left ventricular hypertrophy, in peritoneal dialysis patients.

Clin Nephrol.; 2016, 85(3):135-41.

3- Lee JC, Su SY, Chang, Yang RS, Tsai KS, Collins MT: Characterization of FN1-FGFR1 fusion genes in a large series of phosphaturic mesenchymal tumors. Mod Pathol, 2016, 29(11):1335-46.

4- Mancia G., Fagard R., Narkiewicz K., Redon A Renata C. ESH/ESC Guidelines for the management of arterial hypertension.

European Heart Journal, 2013, 34; 2159-2219.

5- Wanner C, Amann K, Shoji T: The heart and vascular system in CKD"

The Lancet, 2016vol. 388, no. 10041, pp. 276-284.
6- Warady BA and V Chadha: Chronic kidney disease in children: The global perspective Pediatr. Nephrol; 2007, 22 (12):1999-2009.

7- Harambat J, van Stralen KJ, Tizard EJ: Epidemiology of chronic kidney disease in children. Pediatr Nephrol; 2012, 27(3):363-373.

8- Becherucci F, Roperto SR, Materassi M, Romagnani P: Chronic kidney disease in children Clinical Kidney Journal. 2016, vol. 9, no. 4, 583-591.

9- Yilmaz G, Ustundag S, Temizoz O, Sut N, Demir M, Ermis V, Sevinc C, Ustundag A: Fibroblast growth factor 23 and carotid artery intima media thickness in chronic kidney disease. ClinLab.; 2015, 61(8):1061-70. 10- Elshafie A, Bahbah M, Fathia M, Seham M. Ragab, Zein A. Saber O: Carotid intima-media thickness in children with end-stage renal disease on hemodialysis Menoufia Medical Journal. 2016, 29:280-284.

11- Liu D, Alvarez-Elías A, Wile B, Filler G. Deviations from the expected relationship between serum FGF23 and other markers in children with CKD (2017) a crosssectional study BMC Nephrol. 2017 Jun 28; 18(1):204.

References were revised according to the (geget) GLs

\section{Declaration}

Ethics approval and consent to participate

This study protocol and the consents were approved and deemed sufficient by Ethical Committee of Pediatric Department, Faculty of Medicine, Al-Azhar University. And informed written consent was obtained in every case from their legal guardians.

\section{Funding}

The authors declare that they didn't receive any financial support from agencies or others.

\section{Conflict of interest}

There is no conflict of interest regarding the research or publications.

Acknowledgements

We would like to thank all patients and their family members for their valuable contributions to the study. 